Spatial Demography 2012 1(2): 202-218

http://spatialdemography.org

OPEN ACCESS

via Creative Commons 3.0

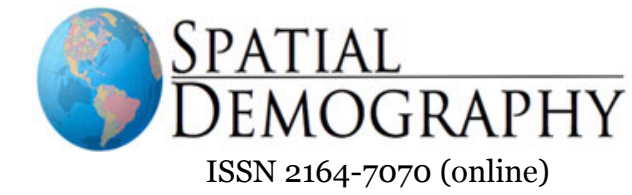

\title{
COLUMN
}

\section{Spatial Sufficiency of 5\% Medicare Standard Analytic Files}

\author{
Lee R. Mobley \\ Editor, Spatial Data
}

\section{ABSTRACT}

The 5\% Medicare Standard Analytic Files (SAF) are random samples used to analyze national trends in medical treatments, expenditures, and outcomes. Their utility in small-area or multilevel analyses is unknown. To demonstrate possible limitations of the $5 \%$ SAF for analysis of health behaviors in small areas. We use descriptive Chi-square goodness-of-fit tests and mapping to explore consistency in the $5 \%$ representation of the $100 \%$ population in states and counties. We conduct multilevel modeling of individual utilization of mammography or endoscopy services for cancer screening and contrast findings across the 5\% and 100\% files. Subjects are enrolled in both parts A and B Medicare coverage and ages 65-104, alive and residing in the same state, with no gaps in coverage during the study period. Identically defined groups are drawn from the $5 \% \mathrm{SAF}$ and $100 \%$ population claims and denominator files. The Chi-square tests of homogeneous population subgroups in 5\% and $100 \%$ files exhibit significant differences in 7 of 8 states. Maps confirm this among states' counties and find that one state is generally under-represented by the $5 \% \mathrm{SAF}$, while others show areas with variable representation. Multilevel modeling results are largely consistent across the partitions of the data, but $5 \%$ sample models have much lower statistical power. Area-level covariate effect estimates show some differences across the two datasets. Multilevel modeling with contextual variables may be misleading in small area analyses conducted using 5\% Medicare SAFs. Provider supply and market characteristics show inconsistent results. Disparities research may benefit from 100\% files to provide statistical power needed to detect meaningful differences. This is significant because the Centers for Medicare and Medicaid Services have recently curtailed permissions to use the $100 \%$ files. These $100 \%$ files are one of few sources of population data available in the U.S. that are representative of small areas in the U.S.. In times of constrained budgets, using population data files is essential so that resources can be targeted to areas robustly identified as having greatest need or gaps in outcomes.

KEYWORDS: Sampling, population health, spatial analysis, access to care, cancer control

\section{INTRODUCTION}

The Centers for Medicare and Medicaid Services (CMS) provide to researchers 5\% sample extracts from claims for reimbursement for Medicare services rendered by various kinds of providers. The 5\% extracts, known as the 5\% Standard Analytic Files (SAF), are randomly drawn from the 100\% population of claims files using a standard set of Health Insurance Claim (HIC) numbers, to provide manageable subsets of the $100 \%$ data files that allow researchers to track a standard cohort of individuals over time.

Many recent studies have used the 5\% Medicare SAF to analyze national trends in medical treatments and outcomes, including: changes 
over time in coronary revascularization procedures, outcomes, and costs (Ryan et al, 2009), outcomes and costs associated with peripheral arterial disease (Jaff et al, 2010), heart failure readmission rates (Aranda et al, 2009), outcomes in heart failure patients after major non-cardiac surgery (Hernandez et al, 2004), glaucoma surgery (Strutton and Walt, 2004), and abdominal aortic aneurysm repair (Dillavou et al, 2006). Other studies have used the SAF to analyze trends in Medicare expenditures by persons receiving different treatment paths. Pyenson et al (2004) used the SAF to examine total expenditures for Medicare patients with terminal medical conditions; Niefeld et al (2003) examined the impact of comorbid conditions on preventable hospitalizations among Medicare beneficiaries aged $65+$ years with type 2 diabetes; and Hogan et al (2001) used the SAF to examine Medicare beneficiaries' expenditures in the last year of life.

The 5\% Medicare SAF is a convenient sample to use in study of national outcomes and statistics, but the sampling design does not ensure that the sample is spatially representative - thus it may not adequately represent the mix of people and services in many small areas of the US. Thus, to understand geographic variation in health care utilization and outcomes, most researchers have used $100 \%$ population files. Examples of this include the Dartmouth Atlas research and other work led by Jack Wennberg (1999), and studies by the Congressional Budget Office (CBO, 2008). Rao et al (2001) used the 100\% Medicare Part B files to study use trends and geographic variation in neuroimaging. Koroukian et al (2005, 2006) used 100\% Medicare population data to examine disparities in use of colorectal cancer screening and spillovers from Medicare managed care onto fee-for-service (FFS) Medicare CRC screening behaviors. Mobley et al (2006) used 100\% Medicare hospital inpatient claims data to examine variation in admission for preventable conditions among the elderly, and Connor et al (2007; 2008) used $100 \%$ claims files to examine geographic variation in hospice use across the US. More recently, Mobley et al (2011) used the 100\% Medicare Part B and denominator files to study diffusion of endoscopy technology over the period 2001-2006.

We know only one study using the $5 \%$ sample to study geographic variations. Pearlman et al (2007) used the 5\% SAF to study geographic variations in the use of echocardiography among Medicare beneficiaries between 1999 and 2004, to determine the rate of growth in these services and evaluate the drivers of growth. A potential problem with this analysis is that the local-area (or even state-level) geographic representativeness of the $5 \%$ file is not known. Thus the findings are likely to be conditional on the sample, and not generalizeable to the entire Medicare population.

While the $5 \%$ sample is a random sample and is expected to be nationally representative of the traditional Medicare beneficiary population, but will not necessarily be representative of this population in smaller, sub-national areas. We use several different analyses to explore the small-area representativeness of the $5 \% \mathrm{SAF}$, using (only) people with traditional Medicare FFS (with complete claims information) in several comparisons. First, we use Chi-square tests of goodness-of-fit to assess whether area proportions by population subgroup are equivalent in the $5 \%$ and $100 \%$ Medicare FFS sample files, at the state level. Next, to explore any patterns in deviations in the 5\% and 100\% proportions at the sub-state level, we map the county-level ratios of $5 \%$ sample counts to $100 \%$ populations, county by county. Maps demonstrate any spatial patterns suggesting clusters of areas with over/under representation of the $100 \%$ population by the $5 \%$ file. Finally, we conduct multilevel modeling of the propensity for individuals to use mammography or endoscopy for breast or colorectal cancer screening or diagnostics. We use the $5 \%$ FFS sample and the $100 \%$ FFS population to estimate the same empirical model, and contrast the findings.

We conclude with a summary of the findings from these three sets of analyses. The main contribution of this paper is to demonstrate some possible limitations of using the 5\% SAF for analysis of population behaviors in counties or smaller areas. 


\section{METHODS}

\section{Sampling Design for the 5\% Medicare Enrollment File}

At age 65 or time of entitling disability, people enroll in Medicare and their personal information becomes part of the Enrollment Data Base (EDB). The 100\% Denominator File is an annually extracted, abbreviated version of the EDB, combining Medicare beneficiary entitlement status information from administrative enrollment records with thirdparty payer information, and information regarding enrollment in Part A (hospitalization), Part B (outpatient care), help with Part B premiums from state Medicaid/state buy-in (MEDPAC, 2004), and enrollment in Medicare HMOs. Every year CMS pulls a 5\% enrollment sample from the Denominator File, based on enrollees having one of the following numerical sequences in positions 8 and 9 of their personal Health Insurance Claim (HIC) number: 05, 20, 45,70 , or 95 . The same numerical sequences in positions 8 and 9 are used in drawing the $5 \%$ file in consecutive years. This 5\% enrollment sample is the basis for the $5 \%$ SAF drawn from the claims files.

While this sampling method produces a random sample that is likely to be nationally representative, there is no guarantee that the sample is spatially random. That is, there is no guarantee that certain areas won't contain disproportionate numbers of persons in the 5\% enrollment and SAF. Thus the $5 \%$ file may not be locally representative, which has implications for any geospatial analyses using the $5 \%$ files. To assess the spatial sufficiency of the Medicare 5\% SAF in utilization and outcomes research requires that we focus our analysis on people with traditional FFS Medicare coverage only. Other groups lack Medicare claims information for use in the comparisons.

\section{Data and Measures}

We used the annual 100\% Medicare denominator files 2001-2005, and annual extracts from 100\% Medicare physician carrier and outpatient claims for all mammography and endoscopy services used 2001-2005. We created a 'pure FFS' sample for each year (2001 to 2005) from the 100\% population iles. The annual 'pure FFS' subjects included beneficiaries aged 65 to 104 , who were alive the entire year, had both Parts A and B Medicare coverage, had no Medicare HMO coverage during the year, and remained living in the same state during the study period (so that a single area context was relevant for the multilevel modeling). We then used the 5\% SAF indicator variable in the denominator file to draw the $5 \%$ subsample from the $100 \%$ population file. We prepared these data files for eight states to use in this analysis (California, Connecticut, Iowa, Kentucky, Louisiana, New Jersey, New Mexico, and Utah).

\section{Statistical Analyses}

We used three methods to assess differences in the $5 \%$ and $100 \%$ files and whether these differences matter for empirical research: 1) Chisquare tests, 2) mapping of ratios of $5 \%$ to $100 \%$ area populations, and 3) multilevel modeling of personal cancer test use.

Chi-square goodness-of-fit tests

We use Chi-Square tests in SAS 9.2 (SAS, 2010) to test homogeneity in the proportions of population subgroups (age, race or ethnicity, gender) across the 5\% and $100 \%$ sample files, at the state level. The null hypothesis for the test is that the proportions of sample members in each state are equivalent in the 5\% and 100\% samples. This hypothesis would not be rejected if the 5\% file was perfectly representative of the $100 \%$ file in every subgroup category in the state. The Chisquare distribution is used to determine how much deviation might occur by chance, and rejection of this hypothesis leads to the conclusion that the 5\% and $100 \%$ files come from significantly different distributions of subgroup population characteristics.

We conduct the test separately for each state and 
Table 1. Chi-square Tests of Goodness of Fit Comparing 5\% and 100\% File Subgroup Proportions, by State for 8 states (statistically significant findings bolded).

\begin{tabular}{lrrrrrrr}
\hline & & \multicolumn{2}{c}{ Race or ethnicity } & \multicolumn{2}{c}{ Sex } & \multicolumn{2}{c}{ Age } \\
\cline { 3 - 8 } \multicolumn{1}{c}{ State } & Year & Chi-square & p-value & Chi-square & p-value & Chi-square & p-value \\
\hline California & 2001 & $\mathbf{4 1 0 . 0 9 8}$ & $\mathbf{0 . 0 0 0}$ & $\mathbf{3 2 . 8 2 3}$ & $\mathbf{0 . 0 0 0}$ & $\mathbf{1 0 . 6 9 7}$ & $\mathbf{0 . 0 0 4 8}$ \\
California & 2003 & $5 \mathbf{0 0 . 6 0 7}$ & $\mathbf{0 . 0 0 0}$ & $\mathbf{3 0 . 2 3 6}$ & $\mathbf{0 . 0 0 0}$ & 3.726 & 0.1552 \\
Connecticut & 2001 & 6.558 & 0.256 & $\mathbf{5 . 6 2 5}$ & $\mathbf{0 . 0 1 8}$ & 2.477 & 0.2898 \\
Connecticut & 2003 & 1.288 & 0.936 & $\mathbf{4 . 3 3 1}$ & $\mathbf{0 . 0 3 7}$ & 1.621 & 0.4446 \\
Iowa & 2001 & $\mathbf{1 2 . 9 3 0}$ & $\mathbf{0 . 0 2 4}$ & $\mathbf{3 . 9 5 4}$ & $\mathbf{0 . 0 4 7}$ & 0.693 & 0.7072 \\
Iowa & 2003 & 5.254 & 0.386 & $\mathbf{4 . 5 8 3}$ & $\mathbf{0 . 0 3 2}$ & 2.157 & 0.3402 \\
Kentucky & 2001 & 4.801 & 0.441 & $\mathbf{2 4 . 8 3 6}$ & $\mathbf{0 . 0 0 0}$ & 1.583 & 0.4533 \\
Kentucky & 2003 & 5.468 & 0.361 & $\mathbf{2 0 . 1 2 4}$ & $\mathbf{0 . 0 0 0}$ & 2.859 & 0.2395 \\
Louisiana & 2001 & $\mathbf{2 1 . 7 6 0}$ & $\mathbf{0 . 0 0 1}$ & $\mathbf{4 3 . 8 8 4}$ & $\mathbf{0 . 0 0 0}$ & $\mathbf{9 . 6 1 4}$ & $\mathbf{0 . 0 0 8 2}$ \\
Louisiana & 2003 & 7.359 & 0.195 & 36.742 & $\mathbf{0 . 0 0 0}$ & 3.017 & 0.2212 \\
New Jersey & 2001 & 10.354 & 0.066 & $\mathbf{2 8 . 2 3 1}$ & $\mathbf{0 . 0 0 0}$ & $\mathbf{1 0 . 8 3}$ & $\mathbf{0 . 0 0 4 4}$ \\
New Jersey & 2003 & $\mathbf{1 3 . 0 4 6}$ & $\mathbf{0 . 0 2 3}$ & $\mathbf{1 7 . 1 0 6}$ & $\mathbf{0 . 0 0 0}$ & $\mathbf{8 . 5 8 9}$ & $\mathbf{0 . 0 1 3 6}$ \\
New Mexico & 2001 & $\mathbf{1 3 2 . 8 7 4}$ & $\mathbf{0 . 0 0 0}$ & 0.077 & 0.781 & 1.326 & 0.5153 \\
New Mexico & 2003 & 1.230 & 0.942 & 0.907 & 0.341 & 0.903 & 0.6366 \\
Utah & 2001 & $\mathbf{2 3 . 7 3 9}$ & $\mathbf{0 . 0 0 0}$ & 2.639 & 0.104 & 0.762 & 0.6832 \\
Utah & 2003 & 4.041 & 0.544 & 0.571 & 0.450 & 0.929 & 0.6286 \\
\hline
\end{tabular}

population subgroup (age, race or ethnicity, gender). The number of classes in the test statistic is the number of classes in the subgroup of interest in the state. For gender, there are 2 classes; for age, there are three classes $\left(65^{-74}, 7^{-}\right.$ $\left.84,85^{+}\right)$and for race or ethnicity there are six classes (white, African American, Hispanic, Asian, Native American, other). We lose 1 degree of freedom because the state population count is used to derive all proportions in the test statistic. The degrees of freedom for the test statistic is (number of classes - 1), so each state has the same degrees of freedom for each subgroup test. A larger value for the test statistic indicates greater discordance between the $5 \%$ and $100 \%$ files at the state level. Results from this analysis are presented in Table 1.

\section{Mapping the $5 \%$ file to $100 \%$ file ratio}

We use mapping to show where within states the $5 \%$ sample is disproportionate to exactly $5 \%$ of the $100 \%$ file. (This discordance results in the statistically significant state-level Chi-square test statistics noted above). To do this, we map the county-level ratio of the $5 \%$ sample counts to $100 \%$ file counts. We use a tri-color map to show counties where the ratio $=1$ ( the $5 \%$ sample is exactly $5 \%$ of the $100 \%$ population sample in the county, colored white), $>1$ (the 5\% sample contains $>5 \%$ of the $100 \%$ population in the county, colored red) and $<1$ (the $5 \%$ sample contains $<5 \%$ of the $100 \%$ population in the county, colored blue). Because the population denominator is so large, the ratio is differentiated across counties at 6 decimal places. To simplify and focus on significant patterns, we round the ratio to 3 decimal points and count anything $\leq$ 0.045 as $<5 \%$, and anything $\geq 0.055$ as $>5 \%$. Results from this analysis are presented in Figure 1.

\section{Multilevel Modeling}

We use the socio-ecological model from previous research to specify a comprehensive set of variables to include in the multilevel model (Mobley et al, 2008a; 2008b; 2010). These include person-level, local area level, and county level variables. We define the health system 
Figure 1. Ratio of 5\% to $100 \%$ file observation counts by county in 8 states, 2001(top) and 2003 (bottom)

2001 data
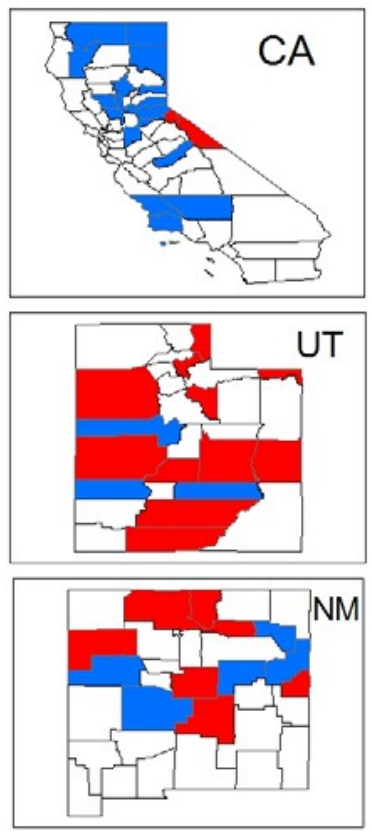

2003 data
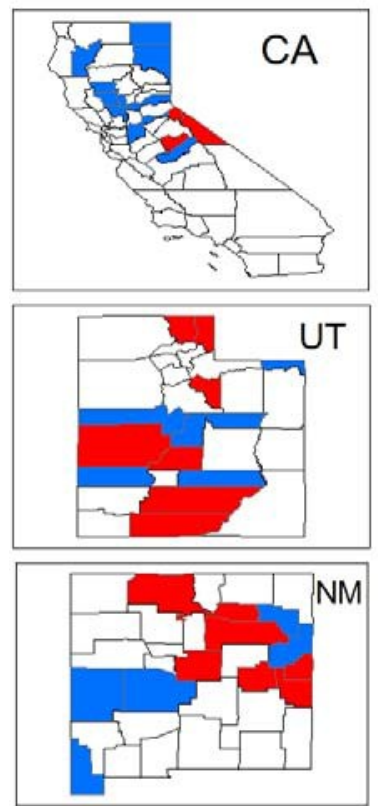
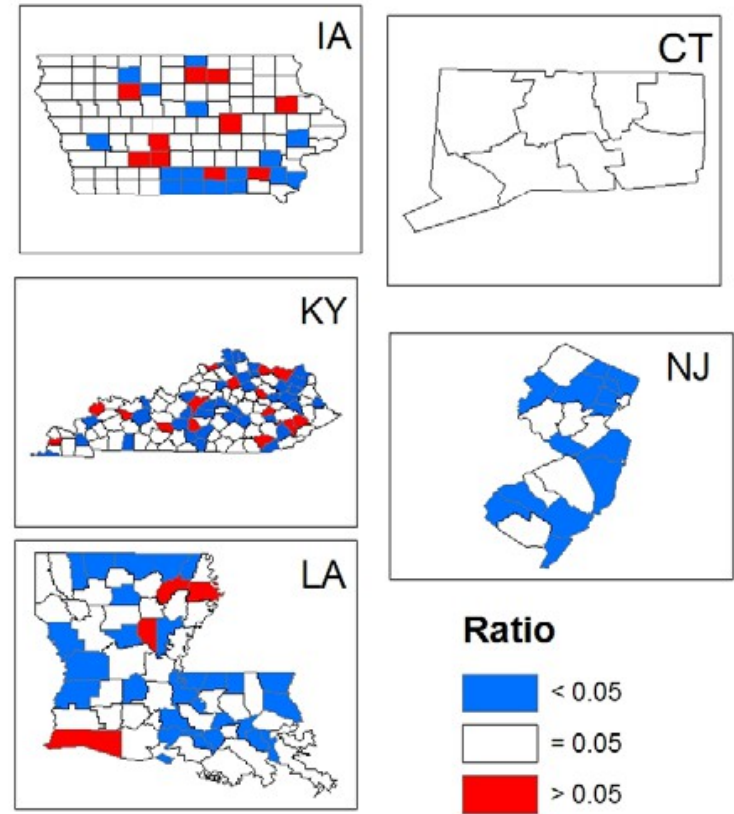

Ratio
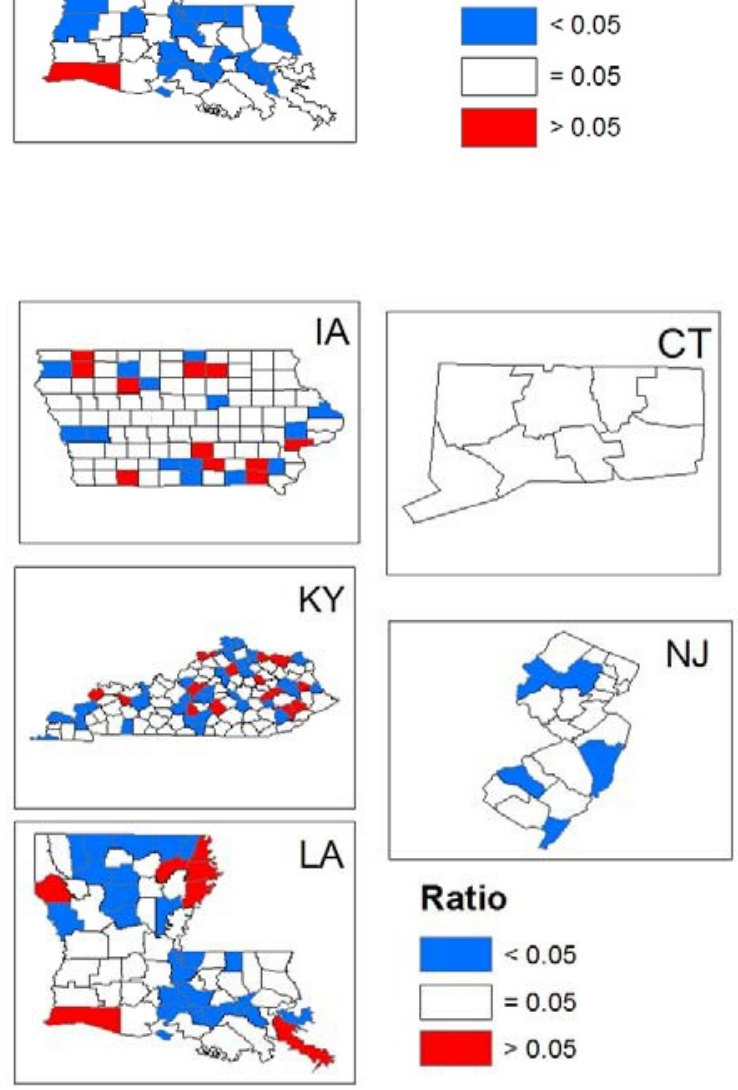

Ratio

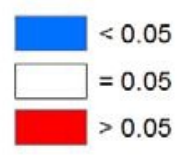


factors at the county level, and these include capacity factors (availability of endoscopy or mammography facilities, and oncologists) and market factors impacting diffusion dynamics (MMC penetration, area poverty). The sociodemographic factors are defined for smaller areas known as primary care service areas (PCSA), which were formed from the aggregation of ZIP code tabulation areas to reflect Medicare patient travel to primary care providers (Goodman et al, 2003). PCSAs are smaller than counties, and are thought to better represent local neighborhood conditions (Mobley et al, 2008a).

For these analyses, the cohort identified in 2001 and satisfying in subsequent years all exclusion criteria (age, coverage, single state residence, death described above) is followed over subsequent years to determine whether any utilization occurred. For mammography (radiograph or MRI), the cohort is followed 3 years (2001-2003). For endoscopy (colonoscopy or sigmoidoscopy) the cohort is followed 5 years (2001-2005). A binary indicator is created if any use occurs over the period.

The multilevel probit model is estimated in SAS. Tables 3 and 4 contain estimated marginal probability effects, i.e. the coefficient estimate '0.05' on covariate $\mathrm{X}$ is interpreted: 'a small change in covariate $\mathrm{X}$ from its mean value is associated with a 5\% higher probability of utilization'. We use Generalized Estimating Equations (GEE) to control for clustering of people within counties so that the county-level variables have robust estimates of their standard errors, and the effect estimates are thus reliable in statistical inference. Results from this analysis are presented in Table 3 (mammography utilization, 2001-2003) and Table 4 (endoscopy utilization, 2001-2005).

\section{RESULTS}

\section{Chi-square Test Findings}

The Chi-square tests of homogeneous population subgroups (Table 1) find the greatest discrepancies in California and in New Mexico, as regards the race or ethnicity subgroups in 2001. By 2003 the difference was reduced in California and nonexistent in New Mexico. In Louisiana, Iowa, and Utah, discrepancies existed in 2001 but disappeared by 2003. Only in New Jersey did the discrepancy in race or ethnicity subgroups not exist in 2001 but become apparent by 2003. In Kentucky and Connecticut, no significant discrepancies existed between the 5\% and 100\% files in terms of racial or ethnic subgroup proportions at the state level, in either period.

All states except New Mexico and Utah exhibited significant discrepancies in the sex proportions in both years. California and Louisiana exhibited discrepancies in the age proportions in 2001 that disappeared by 2003, while New Jersey exhibited discrepancies in both periods. Overall, California and New Jersey exhibited the greatest discordance between the 5\% and 100\% files, with significant differences noted in 5 out of 6 tests. Louisiana exhibited discordance in 4 out of 6 tests; Iowa 3 out of 6 tests; Connecticut, 2 out of 6 and Utah, New Mexico and Kentucky only 1 out of 6 tests. Also, there is more agreement in age than in other subgroup dimensions.

\section{Findings from Mapping}

Maps (Figure 1) are used to demonstrate where among the states' counties discordance exists in the proportion of the $100 \%$ population actually represented by the $5 \% \mathrm{SAF}$. Connecticut is the only state that is apparently well-represented by the 5\% SAF. New Jersey has several underrepresented counties, but no over-represented counties, so the state is generally underrepresented by the $5 \% \mathrm{SAF}$. This may reflect the possibility that many elderly persons leave New Jersey for warmer climates. Other states show a mix of over- and under-represented areas, and the map patterns change over time for all states except Connecticut, and New Jersey is consistently under-represented over time.

\section{Multilevel Regression Findings}

Table 2 contains sample statistics for the interested reader; highlighted rows correspond to the following observations. The $5 \%$ sample is 
Spatial Demography 2012 1(2): 202-218

Mobley, Spatial Data Column

Table 2. Sample Statistics by person, local area PCSA, and county for covariates in multilevel regression of mammography or endoscopy utilization

\begin{tabular}{|c|c|c|c|c|}
\hline State & \multicolumn{2}{|c|}{ California } & \multicolumn{2}{|c|}{ Connecticut } \\
\hline Variable & $5 \%$ File & $100 \%$ File & $5 \%$ File & $100 \%$ File \\
\hline \multirow{2}{*}{$\begin{array}{l}\text { Person level (N) - BC Screening } \\
\text { Ratio N (5\% file) / N (100\% file) }\end{array}$} & 38,769 & 781,832 & 9,208 & 177,375 \\
\hline & \multicolumn{2}{|c|}{0.050} & \multicolumn{2}{|c|}{0.052} \\
\hline Prop. used mammography 2001-2003 & 0.555 & 0.580 & 0.572 & 0.593 \\
\hline age75-84 & $0.385(0.487)$ & $0.385(0.487)$ & $0.408(0.492)$ & $0.405(0.491)$ \\
\hline age 85+ & $0.116(0.321)$ & $0.117(0.321)$ & $0.132(0.338)$ & $0.135(0.341)$ \\
\hline African American & $0.047(0.211)$ & $0.046(0.209)$ & $0.044(0.206)$ & $0.044(0.204)$ \\
\hline Hispanic & $0.051(0.220)$ & $0.049(0.215)$ & $0.007(0.086)$ & $0.008(0.089)$ \\
\hline Asian & $0.085(0.279)$ & $0.080(0.272)$ & $0.006(0.074)$ & $0.005(0.071)$ \\
\hline Native American & $0.004(0.063)$ & $0.001(0.034)$ & $<11^{*}$ & $0.000(0.016)$ \\
\hline Other race/ethnicity & $0.035(0.183)$ & $0.035(0.184)$ & $0.008(0.089)$ & $0.006(0.078)$ \\
\hline disability original reason for Medicare & $0.003(0.053)$ & $0.059(0.235)$ & $0.005(0.072)$ & $0.044(0.206)$ \\
\hline have state buy-in Part B coverage & $27.888(44.180)$ & $27.282(43.836)$ & $10.957(29.800)$ & $10.740(29.489)$ \\
\hline moved to new home & $0.080(0.272)$ & $0.087(0.281)$ & $0.074(0.262)$ & $0.071(0.258)$ \\
\hline miles to closest provider & $2.161(3.963)$ & $2.165(3.964)$ & $1.980(2.573)$ & $1.984(2.607)$ \\
\hline Person level (N) - CRC Screening & 54,536 & $1,126,335$ & 12,581 & 245,186 \\
\hline Ratio N (5\% file) / N (100\% file) & \multicolumn{2}{|c|}{0.048} & \multicolumn{2}{|c|}{0.051} \\
\hline Prop. used endoscopy 2001-2005 & 0.375 & 0.382 & 0.425 & 0.427 \\
\hline female & 0.600 & 0.591 & 0.630 & 0.617 \\
\hline age75-84 & $0.365(0.481)$ & $0.365(0.481)$ & $0.386(0.487)$ & $0.385(0.487)$ \\
\hline age 85+ & $0.072(0.258)$ & $0.073(0.260)$ & $0.080(0.271)$ & $0.081(0.274)$ \\
\hline African American & $0.043(0.202)$ & $0.041(0.199)$ & $0.041(0.198)$ & $0.042(0.200)$ \\
\hline Hispanic & $0.056(0.231)$ & $0.052(0.223)$ & $0.007(0.085)$ & $0.008(0.089)$ \\
\hline Asian & $0.087(0.282)$ & $0.083(0.275)$ & $0.006(0.075)$ & $0.005(0.072)$ \\
\hline Native American & $0.004(0.061)$ & $0.001(0.034)$ & $<11^{*}$ & $0.000(0.015)$ \\
\hline Other race/ethnicity & $0.036(0.187)$ & $0.037(0.189)$ & $0.008(0.087)$ & $0.006(0.080)$ \\
\hline disability original reason for Medicare & $0.003(0.055)$ & $0.064(0.244)$ & $0.005(0.068)$ & $0.050(0.218)$ \\
\hline have state buy-in Part B coverage & 25.428 & 24.717 & $8.455(26.212)$ & $8.141(25.738)$ \\
\hline moved to new home & $0.145(0.352)$ & $0.156(0.363)$ & $0.105(0.306)$ & $0.121(0.326)$ \\
\hline miles to closest provider & $1.378(3.548)$ & $2.185(4.046)$ & $1.032(2.009)$ & $1.914(2.241)$ \\
\hline PCSA level (N) & 337 & 338 & 71 & 71 \\
\hline isolation index - African American & $4.925(7.792)$ & $4.910(7.785)$ & $6.794(10.420)$ & $6.794(10.420)$ \\
\hline isolation index - Hispanic & $31.153(23.614)$ & $31.080(23.617)$ & $7.681(10.848)$ & $7.681(10.848)$ \\
\hline isolation index - Asian & $6.666(8.483)$ & $6.649(8.476)$ & $2.339(1.512)$ & $2.339(1.512)$ \\
\hline isolation index - Native American & $2.257(5.730)$ & $2.260(5.722)$ & $0.407(0.565)$ & $0.407(0.565)$ \\
\hline$\%$ workers who commute $60+$ minutes & $10.223(5.957)$ & 10.207 (5.955) & $8.635(5.996)$ & $8.635(5.996)$ \\
\hline \% elderly with no/poor English & $28.619(16.794)$ & $28.671(16.796)$ & $15.077(9.753)$ & $15.077(9.753)$ \\
\hline proportion county in rural tracts & $27.836(34.204)$ & 27.892(34.169) & $24.396(29.946)$ & $24.396(29.946)$ \\
\hline County level (N) & 58 & 58 & 8 & 8 \\
\hline \% Medicare managed care in 1998 & 23.743(16.927) & $23.743(16.927)$ & $14.059(4.158)$ & $14.059(4.158)$ \\
\hline$\%$ poverty rate & $13.247(4.764)$ & $13.247(4.764)$ & $6.425(1.578)$ & $6.425(1.578)$ \\
\hline mammography facilities per thousand elderly & $0.896(0.508)$ & $0.896(0.508)$ & $0.919(0.255)$ & $0.919(0.255)$ \\
\hline oncologists per thousand elderly & $0.077(0.069)$ & $0.077(0.069)$ & $0.098(0.086)$ & $0.098(0.086)$ \\
\hline
\end{tabular}

* <11: Data suppressed due to confidentiality agreements, because fewer than 11 individuals are in this category. 


\begin{tabular}{|c|c|c|c|c|}
\hline \multirow{2}{*}{$\begin{array}{c}\text { Table 2: Sample Statistics (Cont'd) } \\
\text { Variable }\end{array}$} & \multicolumn{2}{|c|}{ Iowa } & \multicolumn{2}{|c|}{ Kentuck } \\
\hline & $5 \%$ File & $100 \%$ File & $5 \%$ File & $100 \%$ File \\
\hline \multirow{2}{*}{\begin{tabular}{|l|} 
Person level (N) - BC Screening \\
Ratio N (5\% file) / N (100\% file) \\
\end{tabular}} & 10,010 & 197,168 & 10,862 & 217,727 \\
\hline & \multicolumn{2}{|c|}{0.051} & \multicolumn{2}{|c|}{0.050} \\
\hline Prop. used mammography 2001-2003 & 0.577 & 0.593 & 0.522 & 0.55 \\
\hline age75-84 & $0.385(0.487)$ & $0.383(0.486)$ & $0.362(0.481)$ & $0.355(0.478)$ \\
\hline age 85+ & $0.128(0.335)$ & $0.132(0.338)$ & $0.098(0.297)$ & $0.099(0.299)$ \\
\hline African American & $0.009(0.095)$ & $0.008(0.089)$ & $0.051(0.221)$ & $0.047(0.212)$ \\
\hline Hispanic & $<11^{*}$ & $0.001(0.031)$ & $<11^{*}$ & $0.000(0.018)$ \\
\hline Asian & $<11^{*}$ & $0.001(0.035)$ & $<11^{*}$ & $0.001(0.035)$ \\
\hline Native American & $<11^{*}$ & $0.000(0.017)$ & $<11^{*}$ & $0.000(0.010)$ \\
\hline Other race/ethnicity & $0.004(0.060)$ & $0.004(0.062)$ & $0.003(0.057)$ & $0.003(0.057)$ \\
\hline disability original reason for Medicare & $0.005(0.070)$ & $0.039(0.194)$ & $<11^{*}$ & $0.071(0.256)$ \\
\hline have state buy-in Part B coverage & $8.063(26.012)$ & $8.265(26.210)$ & $17.223(36.608)$ & $16.860(36.256)$ \\
\hline moved to new home & $0.038(0.192)$ & $0.046(0.209)$ & $0.043(0.204)$ & $0.050(0.217)$ \\
\hline miles to closest provider & $6.603(7.237)$ & $6.570(7.224)$ & $3.742(5.028)$ & $3.785(5.019)$ \\
\hline Person level (N) - CRC Screening & 13,774 & 274,939 & 0.522 & 0.55 \\
\hline Ratio N (5\% file) / N (100\% file) & \multicolumn{2}{|c|}{$\frac{1}{0.050}$} & \multicolumn{2}{|c|}{$\frac{1}{0.049}$} \\
\hline Prop. used endoscopy 2001-2005 & 0.402 & 0.395 & 0.380 & 0.380 \\
\hline female & 0.619 & 0.611 & 0.631 & 0.612 \\
\hline age75-84 & $0.365(0.481)$ & $0.362(0.481)$ & $0.325(0.469)$ & $0.322(0.467)$ \\
\hline age 85+ & $0.078(0.269)$ & $0.081(0.273)$ & $0.060(0.237)$ & $0.058(0.234)$ \\
\hline African American & $0.009(0.094)$ & $0.007(0.085)$ & $0.047(0.212)$ & $0.044(0.204)$ \\
\hline Hispanic & $0.001(0.030)$ & $0.001(0.034)$ & $0.000(0.018)$ & $0.000(0.020)$ \\
\hline Asian & $0.002(0.040)$ & $0.001(0.035)$ & $0.001(0.032)$ & $0.001(0.034)$ \\
\hline Native American & $0.001(0.024)$ & $0.000(0.016)$ & $0.000(0.014)$ & $0.000(0.010)$ \\
\hline Other race/ethnicity & $0.003(0.059)$ & $0.003(0.059)$ & $0.003(0.058)$ & $0.003(0.052)$ \\
\hline disability original reason for Medicare & $0.005(0.072)$ & $0.048(0.214)$ & $0.002(0.040)$ & $0.096(0.295)$ \\
\hline have state buy-in Part B coverage & $5.936(21.930)$ & $6.155(22.255)$ & $13.757(32.863)$ & $13.400(32.448)$ \\
\hline moved to new home & $0.072(0.259)$ & $0.082(0.275)$ & $0.075(0.263)$ & $0.087(0.282)$ \\
\hline miles to closest provider & $3.661(5.358)$ & $5.648(6.382)$ & $2.803(4.572)$ & $4.339(5.146)$ \\
\hline PCSA level (N) & 225 & 225 & 144 & 145 \\
\hline isolation index - African American & $1.099(3.605)$ & $1.099(3.605)$ & $5.872(9.405)$ & $5.841(9.380)$ \\
\hline isolation index - Hispanic & $2.380(3.753)$ & $2.380(3.753)$ & $1.470(1.380)$ & $1.468(1.375)$ \\
\hline isolation index - Asian & $0.677(0.920)$ & $0.677(0.920)$ & $0.610(0.986)$ & $0.608(0.983)$ \\
\hline isolation index - Native American & $0.385(1.073)$ & $0.385(1.073)$ & $0.390(0.537)$ & $0.393(0.535)$ \\
\hline$\%$ workers who commute $60+$ minutes & $4.869(2.584)$ & $4.869(2.584)$ & $9.334(4.691)$ & $9.382(4.710)$ \\
\hline \% elderly with no/poor English & $7.912(13.637)$ & $7.912(13.637)$ & $8.777(14.937)$ & $8.782(14.885)$ \\
\hline proportion county in rural tracts & $73.918(31.226)$ & 73.918(31.226) & $72.384(29.104)$ & $72.574(29.093)$ \\
\hline County level (N) & 99 & 99 & 120 & 120 \\
\hline \% Medicare managed care in 1998 & $1.760(4.351)$ & $1.760(4.351)$ & $2.136(4.330)$ & $2.136(4.330)$ \\
\hline$\%$ poverty rate & $8.757(1.990)$ & $8.757(1.990)$ & $17.526(6.569)$ & $17.526(6.569)$ \\
\hline mammography facilities per thousand elderly wome & $n 1.114(1.207)$ & $1.114(1.207)$ & $1.138(1.409)$ & $1.138(1.409)$ \\
\hline oncologists per thousand elderly & $0.029(0.092)$ & $0.029(0.092)$ & $0.027(0.087)$ & $0.027(0.087)$ \\
\hline
\end{tabular}

* < 11: Data suppressed due to confidentiality agreements, because fewer than 11 individuals are in this category. 


\begin{tabular}{|c|c|c|c|c|}
\hline Table 2: Sample Statistics (Cont’d) & \multicolumn{2}{|c|}{ Louisiana } & \multicolumn{2}{|c|}{ New Jersey } \\
\hline Variable & 5\% File & $100 \%$ File & 5\% File & $100 \%$ File \\
\hline \multirow{2}{*}{$\begin{array}{l}\text { Person level (N) - BC Screening } \\
\text { N (5\% file) / N (100\% file) }\end{array}$} & 9,011 & 184,005 & 19,394 & 415,796 \\
\hline & \multicolumn{2}{|c|}{0.049} & \multicolumn{2}{|c|}{0.047} \\
\hline Prop. used mammography 2001-2003 & 0.531 & 0.551 & 0.476 & 0.502 \\
\hline age75-84 & $0.352(0.478)$ & $0.360(0.480)$ & $0.400(0.490)$ & $0.402(0.490)$ \\
\hline age $85^{+}$ & $0.101(0.302)$ & $0.103(0.303)$ & $0.114(0.318)$ & $0.117(0.322)$ \\
\hline African American & $0.219(0.413)$ & $0.216(0.412)$ & $0.086(0.281)$ & $0.084(0.278)$ \\
\hline Hispanic & $0.003(0.055)$ & $0.003(0.054)$ & $0.018(0.133)$ & $0.017(0.128)$ \\
\hline Asian & $0.002(0.046)$ & $0.002(0.045)$ & $0.008(0.087)$ & $0.008(0.088)$ \\
\hline Native American & $<11^{*}$ & $0.001(0.024)$ & $0.001(0.024)$ & $0.000(0.016)$ \\
\hline Other race/ethnicity & $0.004(0.059)$ & $0.004(0.066)$ & $0.009(0.092)$ & $0.009(0.092)$ \\
\hline disability original reason for Medicare & $0.003(0.053)$ & $0.063(0.242)$ & $0.002(0.046)$ & $0.052(0.222)$ \\
\hline \%have state buy-in Part B coverage & $22.207(40.507)$ & $21.427(39.805)$ & $11.270(30.666)$ & $11.068(30.425)$ \\
\hline moved to new home & $0.045(0.208)$ & $0.052(0.222)$ & $0.063(0.243)$ & $0.064(0.245)$ \\
\hline miles to closest provider & $5.772(7.591)$ & $5.835(7.535)$ & $1.761(2.728)$ & $1.762(2.741)$ \\
\hline \multirow{2}{*}{\begin{tabular}{|l} 
Person level (N) - CRC Screening \\
N (5\% file) / N (100\% file)
\end{tabular}} & 11,859 & 244,130 & 26,055 & 567,836 \\
\hline & \multicolumn{2}{|c|}{0.049} & \multicolumn{2}{|c|}{0.046} \\
\hline Prop. used endoscopy 2001-2005 & 0.365 & 0.376 & 0.382 & 0.396 \\
\hline female & 0.644 & 0.617 & 0.633 & 0.621 \\
\hline age75-84 & $0.319(0.466)$ & $0.328(0.469)$ & $0.374(0.484)$ & $0.378(0.485)$ \\
\hline age $85+$ & $0.061(0.239)$ & $0.060(0.237)$ & $0.072(0.258)$ & $0.073(0.259)$ \\
\hline African American & $0.206(0.404)$ & $0.194(0.396)$ & $0.080(0.272)$ & $0.079(0.270)$ \\
\hline Hispanic & $0.003(0.054)$ & $0.002(0.050)$ & $0.018(0.134)$ & $0.016(0.127)$ \\
\hline Asian & $0.002(0.046)$ & $0.002(0.044)$ & $0.009(0.092)$ & $0.008(0.091)$ \\
\hline Native American & $0.001(0.033)$ & $0.001(0.025)$ & $0.000(0.021)$ & $0.000(0.016)$ \\
\hline Other race/ethnicity & $0.004(0.062)$ & $0.004(0.063)$ & $0.010(0.099)$ & $0.009(0.096)$ \\
\hline disability original reason for Medicare & $0.003(0.056)$ & $0.084(0.277)$ & $0.002(0.041)$ & $0.057(0.233)$ \\
\hline \%have state buy-in Part B coverage & $18.153(37.069)$ & $16.691(35.668)$ & $8.832(27.154)$ & $8.539(26.738)$ \\
\hline moved to new home & $0.086(0.280)$ & $0.113(0.317)$ & $0.109(0.311)$ & $0.113(0.317)$ \\
\hline miles to closest provider & $3.270(5.864)$ & $4.389(5.926)$ & $0.776(1.654)$ & $1.377(1.952)$ \\
\hline PCSA level (N) & 112 & 112 & 139 & 139 \\
\hline isolation index - African American & $35.646(17.050)$ & $35.646(17.050)$ & $12.629(15.832)$ & $12.629(15.832)$ \\
\hline isolation index - Hispanic & $1.938(1.676)$ & $1.938(1.676)$ & $12.147(14.541)$ & $12.147(14.541)$ \\
\hline isolation index - Asian & $1.087(3.093)$ & $1.087(3.093)$ & $5.302(5.532)$ & $5.302(5.532)$ \\
\hline isolation index - Native American & $1.144(2.791)$ & $1.144(2.791)$ & $0.226(0.234)$ & $0.226(0.234)$ \\
\hline$\%$ workers who commute $60+$ minutes & $10.297(4.436)$ & $10.297(4.436)$ & $12.977(5.343)$ & $12.977(5.343)$ \\
\hline$\%$ elderly with no/poor English & $8.398(10.431)$ & $8.398(10.431)$ & $24.459(13.660)$ & $24.459(13.660)$ \\
\hline proportion county in rural tracts & $54.449(30.887)$ & 54.449 (30.887) & 12.445 (22.899) & 12.445 (22.899) \\
\hline County level (N) & 64 & 64 & 21 & 21 \\
\hline \% Medicare managed care in 1998 & $11.961(11.472)$ & $11.961(11.472)$ & $14.000(5.759)$ & $14.000(5.759)$ \\
\hline$\%$ poverty rate & $20.127(5.754)$ & $20.127(5.754)$ & $7.600(3.387)$ & $7.600(3.387)$ \\
\hline mammography facilities per thousand elderly women & $0.545(0.692)$ & $0.545(0.692)$ & $0.944(0.384)$ & $0.944(0.384)$ \\
\hline oncologists per thousand elderly & $0.029(0.068)$ & $0.029(0.068)$ & $0.085(0.067)$ & $0.085(0.067)$ \\
\hline
\end{tabular}

* < 11: Data suppressed due to confidentiality agreements, because fewer than 11 individuals are in this category. 


\begin{tabular}{|c|c|c|c|c|}
\hline Table 2: Sample Statistics (Cont’d) & \multicolumn{2}{|c|}{ New Mexico } & \multicolumn{2}{|c|}{ Utah } \\
\hline Variable & $5 \%$ File & $100 \%$ File & $5 \%$ File & $100 \%$ File \\
\hline $\begin{array}{l}\text { Person level (N) - BC Screening } \\
\text { N (5\% file) / N (100\% file) }\end{array}$ & \multicolumn{2}{|c|}{0.051} & \multicolumn{2}{|c|}{0.052} \\
\hline Prop. used mammography services 2001-2003 & 0.526 & 0.545 & 0.572 & 0.578 \\
\hline age75-84 & $0.334(0.472)$ & $0.340(0.474)$ & $0.361(0.480)$ & $0.363(0.481)$ \\
\hline age $85+$ & $0.091(0.288)$ & $0.096(0.295)$ & $0.090(0.286)$ & $0.093(0.291)$ \\
\hline African American & $0.013(0.114)$ & $0.013(0.112)$ & $0.004(0.063)$ & $0.003(0.057)$ \\
\hline Hispanic & $0.089(0.285)$ & $0.088(0.284)$ & $0.008(0.091)$ & $0.008(0.087)$ \\
\hline Asian & $<11^{*}$ & $0.002(0.047)$ & $0.006(0.080)$ & $0.007(0.081)$ \\
\hline Native American & $0.047(0.212)$ & $0.029(0.168)$ & $0.004(0.063)$ & $0.002(0.045)$ \\
\hline Other race/ethnicity & $0.009(0.094)$ & $0.023(0.149)$ & $0.010(0.099)$ & $0.009(0.094)$ \\
\hline disability original reason for Medicare & $0.004(0.066)$ & $0.052(0.223)$ & $0.004(0.064)$ & $0.039(0.193)$ \\
\hline have state buy-in Part B coverage & $17.689(36.882)$ & $17.831(36.976)$ & $5.328(21.510)$ & $5.363(21.430)$ \\
\hline moved to new home & $0.076(0.265)$ & $0.067(0.249)$ & $0.060(0.237)$ & $0.063(0.243)$ \\
\hline miles to closest provider & $7.947(13.280)$ & $8.064(13.356)$ & $4.785(9.172)$ & $4.743(8.963)$ \\
\hline Person level (N) - CRC Screening & 5,070 & 100,328 & 5,699 & 113,066 \\
\hline $\mathrm{N}$ (5\% file) / N (100\% file) & \multicolumn{2}{|c|}{0.051} & \multicolumn{2}{|c|}{0.050} \\
\hline Prop. used endoscopy 2001-2005 & 0.339 & 0.342 & 0.405 & 0.418 \\
\hline female & 0.574 & 0.570 & 0.589 & 0.578 \\
\hline age75-84 & $0.302(0.459)$ & $0.312(0.463)$ & $0.344(0.475)$ & $0.341(0.474)$ \\
\hline age 85+ & $0.057(0.233)$ & $0.057(0.232)$ & $0.063(0.242)$ & $0.058(0.233)$ \\
\hline African American & $0.012(0.110)$ & $0.012(0.110)$ & $0.004(0.059)$ & $0.003(0.056)$ \\
\hline Hispanic & $0.095(0.293)$ & $0.095(0.293)$ & $0.010(0.098)$ & $0.008(0.088)$ \\
\hline Asian & $<11^{*}$ & $0.002(0.044)$ & $0.006(0.076)$ & $0.007(0.081)$ \\
\hline Native American & $0.052(0.221)$ & $0.031(0.173)$ & $0.004(0.065)$ & $0.002(0.046)$ \\
\hline Other race/ethnicity & $0.010(0.102)$ & $0.022(0.147)$ & $0.010(0.099)$ & $0.009(0.094)$ \\
\hline disability original reason for Medicare & $0.004(0.063)$ & $0.075(0.263)$ & $0.005(0.067)$ & $0.047(0.211)$ \\
\hline \%have state buy-in Part B coverage & $14.499(33.656)$ & $14.950(34.011)$ & $4.330(19.060)$ & $3.927(18.078)$ \\
\hline moved to new home & $0.112(0.315)$ & $0.106(0.308)$ & $0.102(0.303)$ & $0.112(0.316)$ \\
\hline miles to closest provider & $5.245(10.311)$ & $7.271(11.327)$ & $2.040(5.711)$ & $3.834(6.303)$ \\
\hline PCSA level (N) & 61 & 61 & 52 & 54 \\
\hline isolation index - African American & $1.444(1.788)$ & $1.444(1.788)$ & $0.620(1.376)$ & $0.608(1.352)$ \\
\hline isolation index - Hispanic & $50.501(21.295)$ & $50.501(21.295)$ & $9.292(9.350)$ & $9.237(9.205)$ \\
\hline isolation index - Asian & $0.652(0.841)$ & $0.652(0.841)$ & $0.991(1.133)$ & $0.964(1.120)$ \\
\hline isolation index - Native American & $13.889(27.187)$ & $13.889(27.187)$ & $5.894(17.633)$ & $5.706(17.324)$ \\
\hline$\%$ workers who commute $60+$ minutes & $9.317(6.804)$ & $9.317(6.804)$ & $7.176(4.150)$ & $7.156(4.072)$ \\
\hline$\%$ elderly with no/poor English & $24.657(18.552)$ & $24.657(18.552)$ & $20.343(22.459)$ & $20.275(22.039)$ \\
\hline proportion county in rural tracts & $61.932(35.917)$ & $61.932(35.917)$ & $49.856(38.906)$ & $50.094(39.117)$ \\
\hline County level (N) & 33 & 33 & 28 & 29 \\
\hline \% Medicare managed care in 1998 & $7.723(12.256)$ & $7.723(12.256)$ & $5.431(5.834)$ & $5.339(5.750)$ \\
\hline$\%$ poverty rate & $21.091(5.954)$ & $21.091(5.954)$ & $11.650(4.069)$ & $11.576(4.016)$ \\
\hline mammography facilities per thousand elderly women & $0.617(0.758)$ & $0.617(0.758)$ & $1.129(1.606)$ & $1.090(1.591)$ \\
\hline oncologists per thousand elderly & $0.019(0.051)$ & $0.019(0.051)$ & $0.020(0.053)$ & $0.020(0.052)$ \\
\hline
\end{tabular}

$*<11$ : Data suppressed due to confidentiality agreements, because fewer than 11 individuals are in this category. 


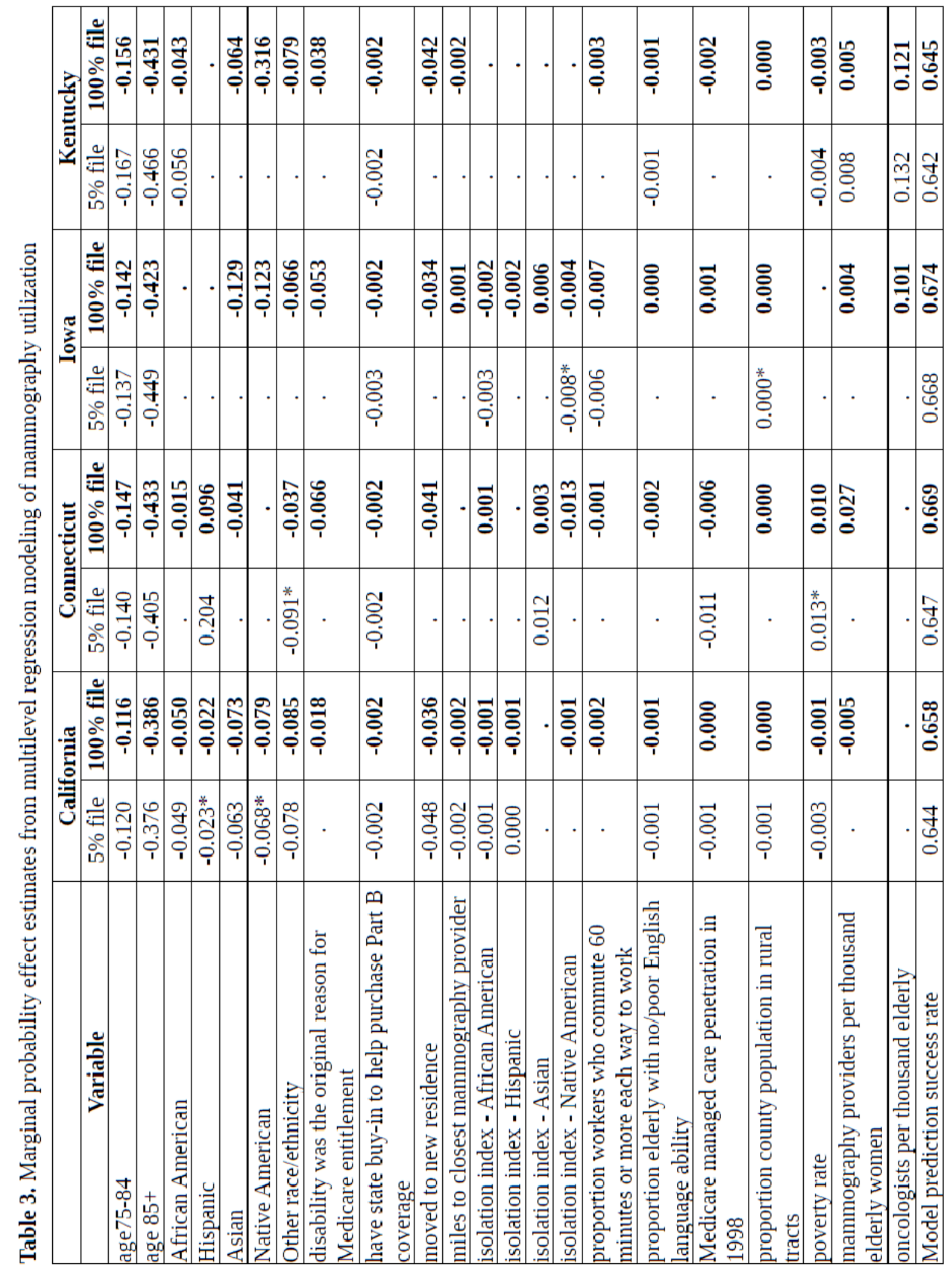




\begin{tabular}{|c|c|c|c|c|c|c|c|c|c|c|c|c|c|c|c|c|c|c|c|c|c|c|}
\hline 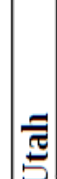 & $\begin{array}{l}0 \\
0 \\
0 \\
0 \\
0 \\
0\end{array}$ & 它 & 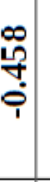 & & &. & î & 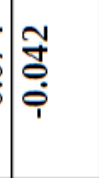 & ָै & 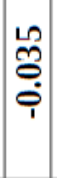 & $\begin{array}{l}0 \\
0 \\
0 \\
0 \\
1\end{array}$ & & & 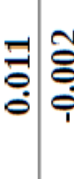 & . & 令 & • & 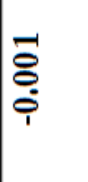 & $\begin{array}{l}1 \\
0 \\
0 \\
0\end{array}$ & بَّ & & \\
\hline & 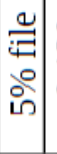 & \begin{tabular}{c}
$\stackrel{1}{7}$ \\
\hdashline \\
\hdashline
\end{tabular} & $\begin{array}{l}0 \\
0 \\
m \\
0 \\
1 \\
1\end{array}$ & & & & . & . & 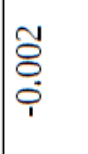 & & $\begin{array}{l}1 \\
0 \\
0 \\
1 \\
1\end{array}$ & & & $\begin{array}{l} \pm \\
8 \\
0 \\
1\end{array}$ & '? & 童 & . & . & $\begin{array}{l}1 \\
0 \\
0 \\
0\end{array}$ & 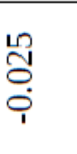 & & \\
\hline 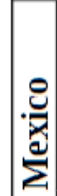 & 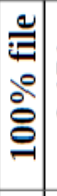 & 它 & 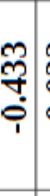 & 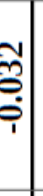 & 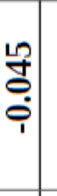 & $\frac{2}{2}$ & $\frac{1}{9}$ & 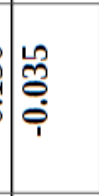 & ฮิ & 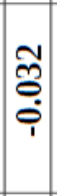 & $\begin{array}{c}-1 \\
\\
0 \\
\\
1\end{array}$ & & - & ఏ & 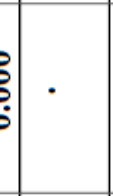 & ஓ̊: & - & 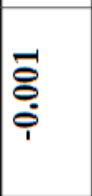 & $\mid \begin{array}{l}0 \\
0 \\
0 \\
0 \\
1\end{array}$ & $\stackrel{0}{0}$ & & \\
\hline 己 & 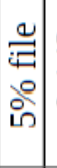 & 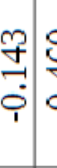 & $\begin{array}{l}0 \\
0 \\
0 \\
0 \\
1\end{array}$ & & $\begin{array}{l}0 \\
1 \\
0 \\
0 \\
1\end{array}$ & $\frac{\text { 의 }}{9}$ & & $\cdot$ & ठ̊ & & & & . & & . & . & . & . & $\begin{array}{l}0 \\
8 \\
0 \\
0 \\
1\end{array}$ & . & $\begin{array}{l}\mathscr{m} \\
m \\
m \\
0\end{array}$ & \\
\hline 总 & 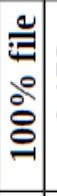 & : & 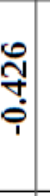 & & 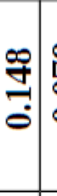 & 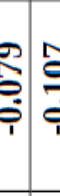 & 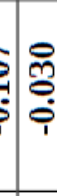 & 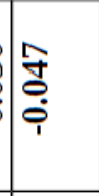 & 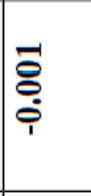 & 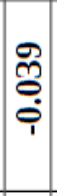 & & 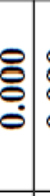 & 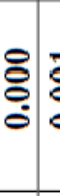 & $\begin{array}{l}-1 \\
\\
0\end{array}$ & . & 告 & ֶิ & ఏ్రి & 苟 & ஜே. & $\frac{9}{9}$ & \\
\hline z & 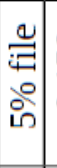 & 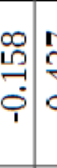 & $\begin{array}{l}\hat{y} \\
y \\
\dot{y} \\
i\end{array}$ & & 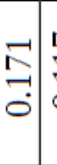 & $\begin{array}{l} \\
\\
\end{array}$ & & 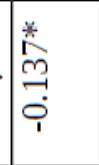 & 8 & $\begin{array}{c}0 \\
0 \\
0 \\
0 \\
i\end{array}$ & & & $\begin{array}{l}\overrightarrow{8} \\
\dot{0}\end{array}$ & & $\cdot \mid \begin{array}{l}m \\
0\end{array}$ & 8 & Oి & & $\mid \begin{array}{c}\text { ma } \\
0 \\
0 \\
0\end{array}$ & & 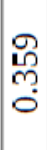 & \\
\hline . & $\begin{array}{l}0 \\
0 \\
0 \\
0 \\
0 \\
0 \\
0\end{array}$ & 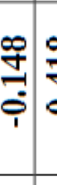 & \begin{tabular}{l}
$\infty$ \\
\multirow{7}{7}{} \\
$\vdots$ \\
\end{tabular} & & . & $\frac{E}{1}$ & م̂ & 导 & $\overline{8}$ & $\mid \begin{array}{l}0 \\
0 \\
\grave{1} \\
1\end{array}$ & 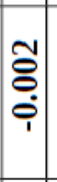 & 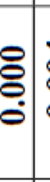 & 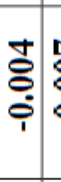 & 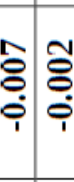 & 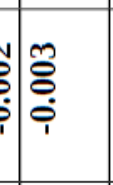 & - & ְִ & 8 & 令 & בְ & & \\
\hline ప్ & 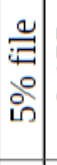 & 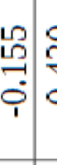 & $\begin{array}{l}0 \\
y \\
⿱ \\
i \\
i\end{array}$ & & & & 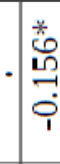 & . & 8 & $\mid \begin{array}{l}3 \\
\mathscr{6} \\
0 \\
1\end{array}$ & $\begin{array}{l}2 \\
0 \\
0 \\
0 \\
1\end{array}$ & & & 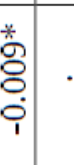 & ${ }^{\circ}$ & . & . & . & 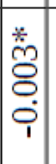 & & & \\
\hline & $\begin{array}{l}\frac{0}{0} \\
\frac{\pi}{7} \\
\frac{\pi}{7}\end{array}$ & 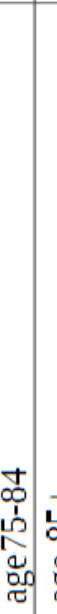 & $\begin{array}{c}+ \\
\text { 岕 } \\
\infty \\
\text { व. } \\
\text { व. }\end{array}$ & 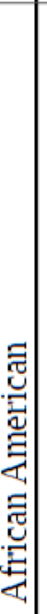 & 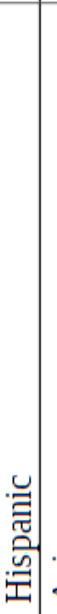 & 壳 & 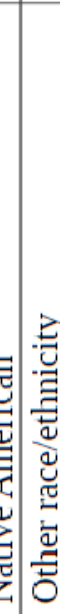 & 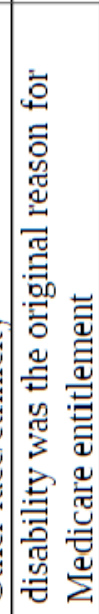 & 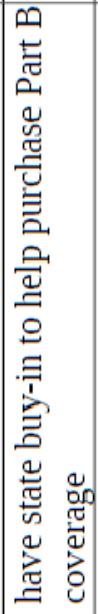 & 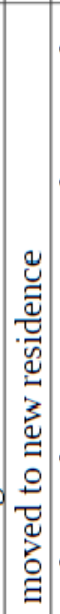 & 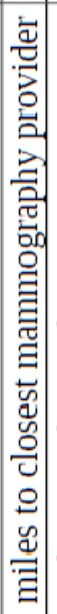 & 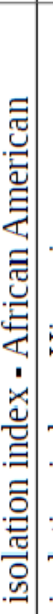 & 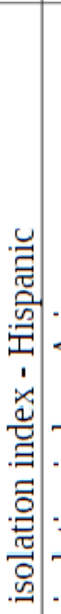 & 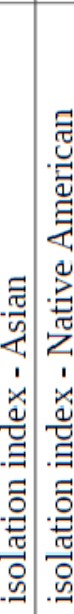 & 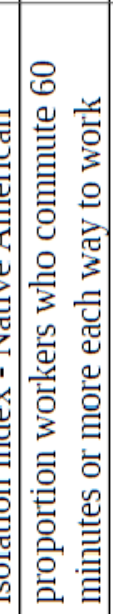 & 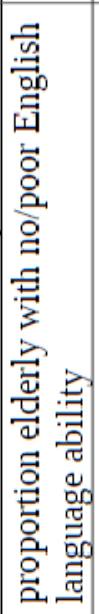 & 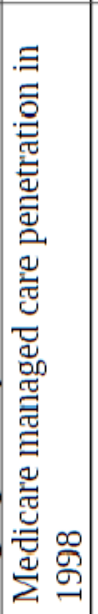 & 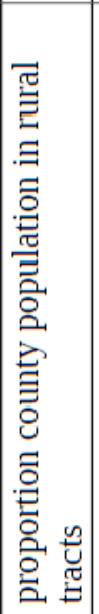 & 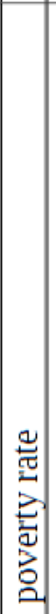 & 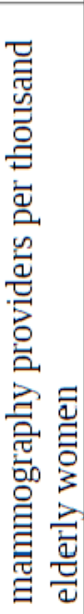 & 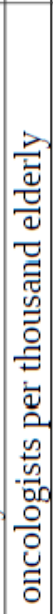 & 曷 \\
\hline
\end{tabular}




\begin{tabular}{|c|c|c|c|c|c|c|c|c|c|c|c|c|c|c|c|c|c|c|c|c|c|c|c|c|c|}
\hline $\bar{z}$ & $\begin{array}{l}0 \\
0 \\
0 \\
0 \\
0\end{array}$ & $\begin{array}{l}0 \\
0 \\
0 \\
\vdots \\
1 \\
\end{array}$ & 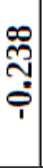 & 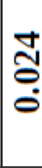 & 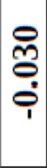 & & & $\begin{array}{l}\vec{Z} \\
\text { స్ } \\
\grave{1}\end{array}$ & & $\begin{array}{l}\Delta \\
\\
\\
\end{array}$ & 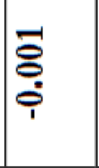 & & $\begin{array}{l}-1 \\
0 \\
0 \\
0 \\
\end{array}$ & $\begin{array}{l}-1 \\
\\
\\
\\
\end{array}$ & 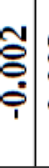 & $\begin{array}{l}\tilde{O} \\
\tilde{\theta} \\
\dot{0}\end{array}$ & & 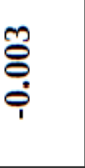 & 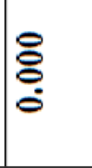 & 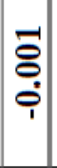 & 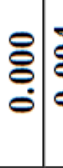 & $\begin{array}{c}\stackrel{0}{0} \\
\\
\end{array}$ & & & \\
\hline & 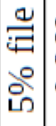 & $\begin{array}{l}0 \\
0 \\
0 \\
\vdots \\
1\end{array}$ & $\begin{array}{l}0 \\
\vdots \\
\sim \\
\vdots \\
1\end{array}$ & $\begin{array}{l}\stackrel{+}{\sim} \\
\stackrel{0}{\circ}\end{array}$ & & & & & & $\cdot$ & 공 & & & $\begin{array}{l}-1 \\
\overline{8} \\
0 \\
0 \\
1\end{array}$ & & $\begin{array}{l}\stackrel{*}{*} \\
\vec{G} \\
0 \\
0\end{array}$ & & ${ }^{\circ}$ & - & & & \begin{tabular}{l} 
L \\
\hdashline \\
\hdashline \\
\hdashline
\end{tabular} & & & $\dot{0}$ \\
\hline & 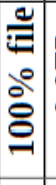 & $\begin{array}{l}0 \\
0 \\
0 \\
0 \\
\\
1\end{array}$ & 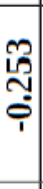 & بூ & & & 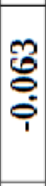 & & $\begin{array}{l}\overrightarrow{0} \\
. \\
\\
\end{array}$ & ฮై & & 의 & 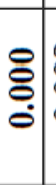 & 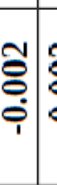 & $\begin{array}{l}2 \\
0 \\
0 \\
0 \\
0\end{array}$ & $\begin{array}{l}\widehat{y} \\
\stackrel{0}{0}\end{array}$ & & $\begin{array}{l}\stackrel{9}{0} \\
\stackrel{0}{0} \\
\end{array}$ & 苛 & 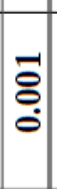 & 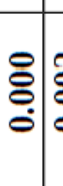 & 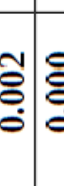 & & & \\
\hline & 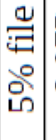 & $\begin{array}{l}0 \\
0 \\
0 \\
0 \\
1\end{array}$ & 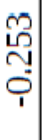 & & & & $\begin{array}{l}0 \\
0 \\
+ \\
0 \\
0\end{array}$ & & $\begin{array}{l}\text { बे } \\
\text { ने } \\
\\
\end{array}$ & $\cdot$ & 范 & & & & | & $\begin{array}{l}\infty \\
0 \\
0 \\
0 \\
0\end{array}$ & & $\begin{array}{l}0 \\
0 \\
0 \\
0 \\
1\end{array}$ & ${ }^{\circ}$ & & & & & & \\
\hline 包 & $\begin{array}{l}0 \\
0 \\
0 \\
0 \\
0 \\
0 \\
0\end{array}$ & $\begin{array}{l}\mathcal{Z} \\
\exists \\
\vdots \\
0 \\
1\end{array}$ & 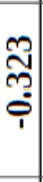 & $\begin{array}{l}\text { Pे } \\
\text { Oें } \\
\text { i }\end{array}$ & & & 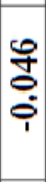 & & $\begin{array}{l}\text { ले } \\
0 \\
0 \\
\\
1\end{array}$ & $\begin{array}{l}\text { 몽 } \\
\text { 웅 }\end{array}$ & & $\begin{array}{l}0 \\
0 \\
\dot{c} \\
\dot{c}\end{array}$ & & $\begin{array}{l}-1 \\
\overline{0} \\
0 \\
\\
1\end{array}$ & & $\begin{array}{l}\stackrel{2}{\circ} \\
\stackrel{0}{0}\end{array}$ & & ֻृ & 응 & & $\begin{array}{l}0 \\
0 \\
0 \\
0\end{array}$ & & & & \\
\hline & 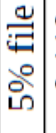 & 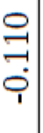 & 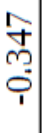 & $\begin{array}{l}\vec{m} \\
0 \\
0 \\
\end{array}$ & & & 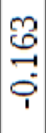 & & & - & 芌 & 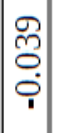 & & 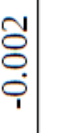 & & $\begin{array}{l}0 \\
\stackrel{2}{0} \\
0 \\
0\end{array}$ & & $\begin{array}{l}\text { Oे } \\
0\end{array}$ & - & 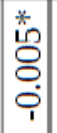 & & & & & \\
\hline త్ర & \begin{tabular}{|c|} 
\\
0 \\
0 \\
0 \\
0 \\
0 \\
0 \\
0
\end{tabular} & 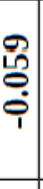 & 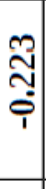 & إْ & $\begin{array}{l}\stackrel{2}{1} \\
\text { ơ } \\
\text { î }\end{array}$ & $\begin{array}{l}\text { 눙 } \\
\text { 응 } \\
\text { †े }\end{array}$ & 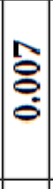 & $\mid \begin{array}{c}0 \\
0 \\
0 \\
0 \\
1\end{array}$ & 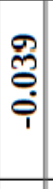 & $\stackrel{0}{\frac{0}{0}}$ & 宮 & 음 & $\begin{array}{l}\overrightarrow{1} \\
0 \\
\\
\end{array}$ & & $\begin{array}{l}\overline{0} \\
\\
\\
\end{array}$ & 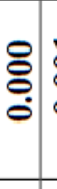 & $\begin{array}{l}0 \\
\\
\\
\\
\end{array}$ & 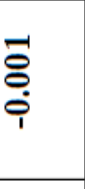 & i̊ & 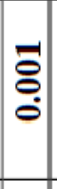 & 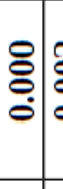 & & & & \\
\hline & 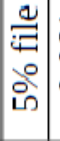 & $\begin{array}{l}\vec{b} \\
0 \\
0 \\
1\end{array}$ & $\begin{array}{l}0 \\
\stackrel{ }{1} \\
\underline{i}\end{array}$ & 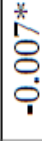 & 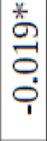 & 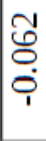 & & $\mid \begin{array}{l}0 \\
\dot{D} \\
0 \\
\dot{1}\end{array}$ & $\begin{array}{l}\text { गे } \\
0 \\
0 \\
\end{array}$ & - & 롱 & & $\begin{array}{l}-1 \\
8 \\
0 \\
0 \\
1\end{array}$ & & $\begin{array}{l}- \\
8 \\
0 \\
0\end{array}$ & 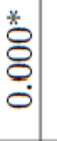 & & • & $\begin{array}{l}0 \\
8 \\
0 \\
\end{array}$ & & 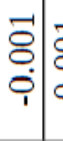 & $\begin{array}{c}-\vec{\delta} \\
\stackrel{0}{0} \\
\dot{0}\end{array}$ & & & \\
\hline & 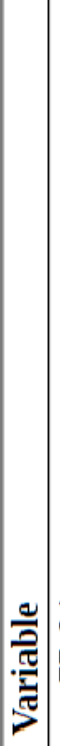 & 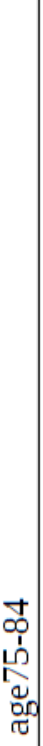 & 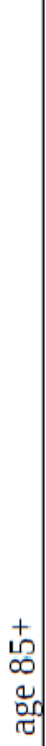 & 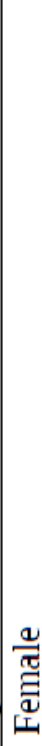 & 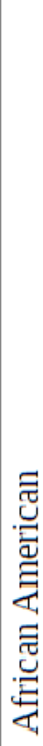 & 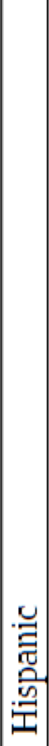 & 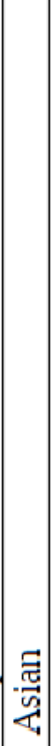 & 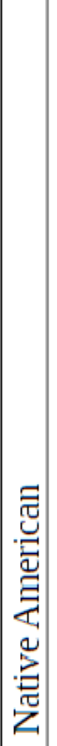 & $\begin{array}{l} \\
\\
\\
\\
\end{array}$ & 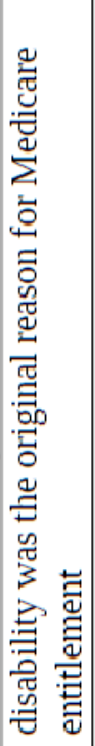 & 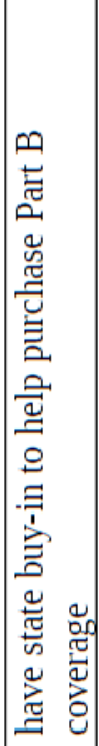 & 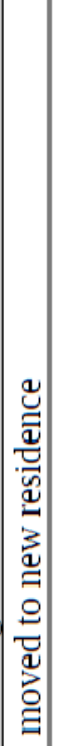 & 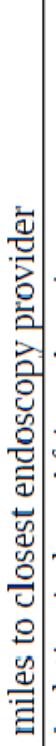 & 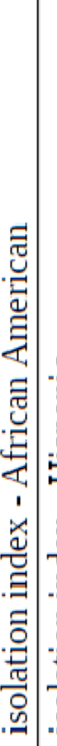 & 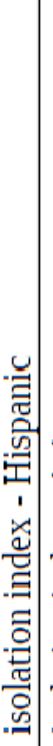 & 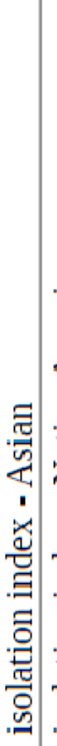 & 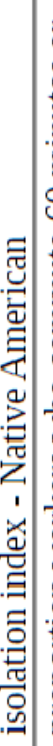 & 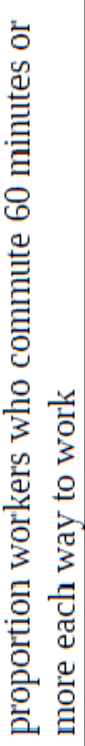 & 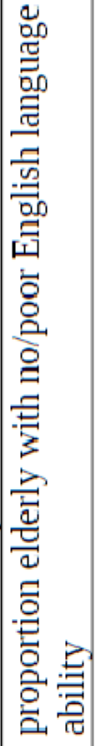 & 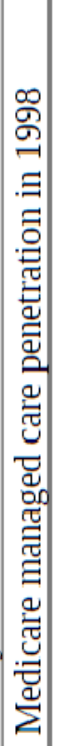 & 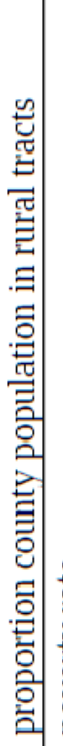 & 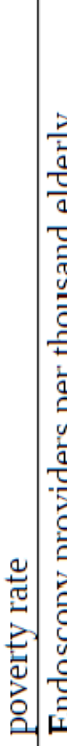 & 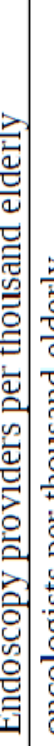 & 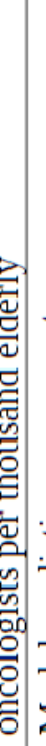 & 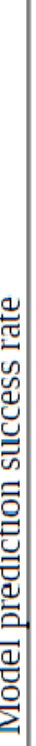 \\
\hline
\end{tabular}




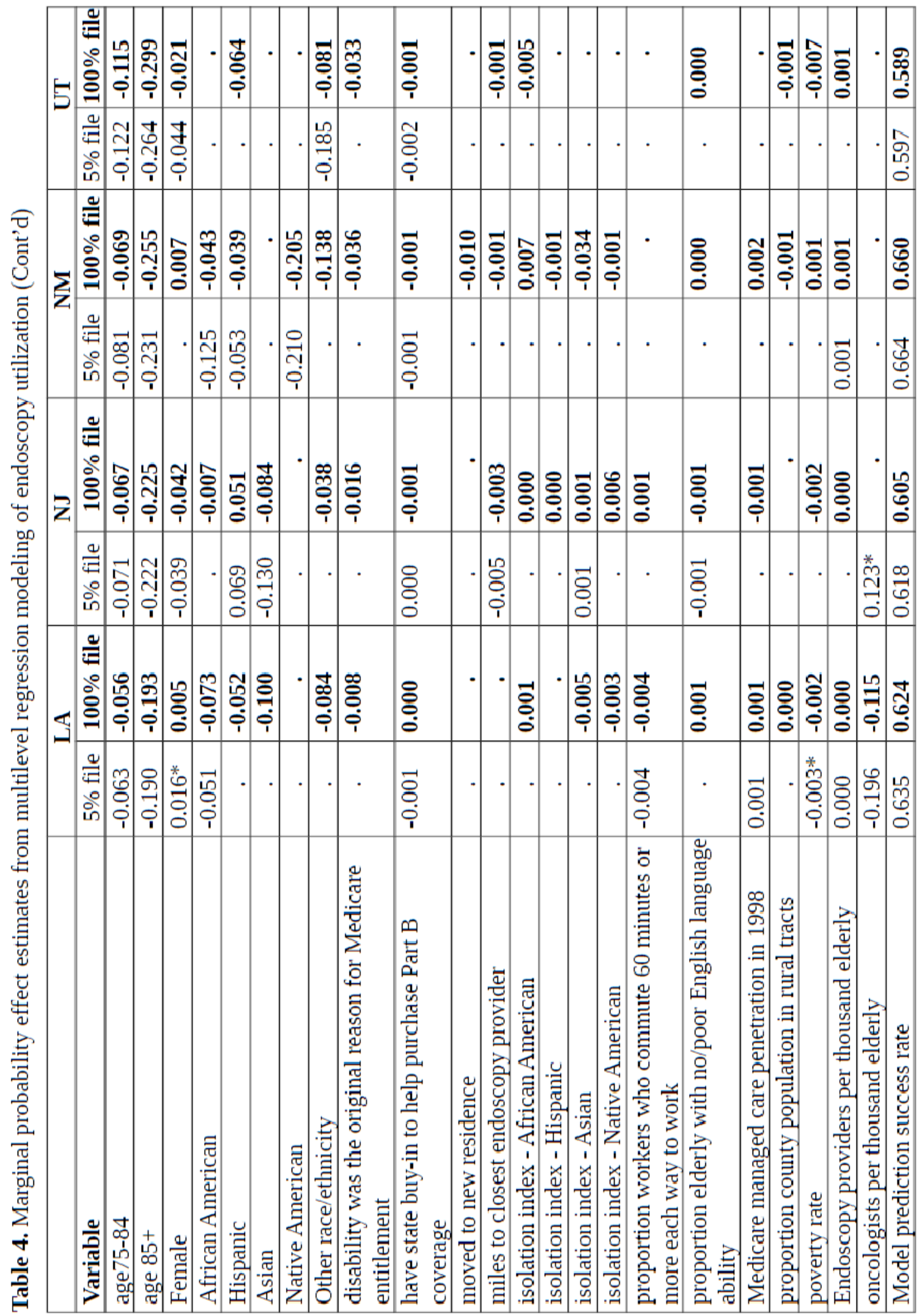


about $5 \%$ as large as the $100 \%$ sample in every state, with the exception of New Jersey, where the $5 \%$ file is several percentage points less than $5 \%$. In Table 2 we also see that the sample proportion who used any mammography 2001-2003 is always lower in the $5 \%$ file than in the $100 \%$ file, thus the $5 \%$ sample is probably not a good predictor of national mammography use rates. Also, the sample proportion who used endoscopy is usually lower in the $5 \%$ file than in the $100 \%$ file (the exceptions being in Iowa and Kentucky). The endoscopy use samples are larger than the mammography use samples, because they include both men and women. The sample statistics also show that the number of counties and local neighborhoods (PCSAs) covered by the two samples are largely equivalent. Exceptions include Utah, where the 5\% sample actually misses 1 county and 2 PCSAs, and in Kentucky and California where it misses 1 PCSA. These areas not covered by the $5 \%$ file are the most sparsely populated counties and PCSAs in these states.

In each Table 3 and 4 , the $5 \%$ sample regression results are presented next to the $100 \%$ sample regression results for each state. Model prediction success rates (bottom row) are roughly the same across the 5\% and 100\% sample regressions. Only the effect estimates that are statistically significant at the $5 \%$ level are presented in the tables. These are augmented by results significant at the $10 \%$ level, indicated with an asterisk, for the $5 \%$ sample model.

Comparisons across the $5 \%$ and $100 \%$ regression results lead to the following conclusions. The person-level variables (age through miles to closest provider) are largely consistent across the two regressions. However, the 100\% sample regression has greater statistical power than the $5 \%$ sample to detect significant effect estimates for sparser population subgroups. For area-level covariates (isolation index through oncologists) the greater power in the $100 \%$ file model is even more evident. Overall, when significant, the 5\% sample results are generally consistent with the $100 \%$ sample results in terms of sign of effect (positive, negative) and often in terms of magnitude of effect. The supply variables (mammography or endoscopy providers per thousand elderly, oncologists per thousand elderly) are generally significant in the $100 \%$ file analysis, but rarely so in the 5\% file analysis.

Cases where the $5 \%$ and $100 \%$ file regression models provide inconsistent findings are shaded green in Tables 3 and 4 . Out of 176 possible pairs of effect estimates ( 22 covariates in 8 states) there are 9 instances where the sign is contradictory or the $5 \%$ model produced a significant effect and the 100\% model did not. Thus about $5 \%$ of the pairs of effect estimates (9 out of 176 cases) are contradictory across the two samples. Four of the disagreements are in California, two are in New Jersey, and one each are in New Mexico Louisiana, and Connecticut. Two of the instances (New Mexico and New Jersey) result from the $5 \%$ model finding a significant and positive effect of oncologist density, while the $100 \%$ model finds none. Two (in Louisiana and New Jersey) are opposite signs on the association between having state buy-in assistance for purchase of part B (low income). The remaining 5 instances (California/4 and Connecticut /1) are associated with percent rural or Medicare managed care penetration.

Unfortunately, all of these (9 out of 176) cases are associated with variables that are of policy importance, and modifiable by policy intervention. However, the disagreements are infrequent overall.

\section{DISCUSSION}

The Medicare 5\% Standard Analytic Files (SAF) may have important limitations in small are analysis due to the sampling method used to construct it, which was not designed to be representative of people in small areas, such as counties. The literature provides no evidence to date regarding whether the $5 \%$ SAF is representative of state samples, or whether using the $5 \% \mathrm{SAF}$ to model neighborhood influences in multilevel modeling is misleading due to nonrepresentation of areas by the 5\% sample. This paper is the first to demonstrate potential limitations of Medicare 5\% FFS SAF regarding 
small- area sufficiency of these data when used in spatial analysis of access and utilization of healthcare services.

We used the 5\% SAF indicator provided in Medicare denominator file, and focused on 8 states. We fond that the $5 \%$ and $100 \%$ file samples are generally consistent in terms of empirical results from multilevel regression, although statistical power is greater in the $100 \%$ file and a greater number of covariates have significant estimates.

The two states where the instances of discordance between the $5 \%$ and $100 \%$ regressions are most numerous are also the two states with the greatest discordance exhibited from the Chisquare tests - California and New Jersey. The Chi-square analysis tests the assumption that the distribution of population subgroups is equal in the two partitions of the data $(100 \%$ versus $5 \%$ files) for a particular state. In New Jersey, the $5 \%$ file is also 2-3 percentage points smaller than $5 \%$ of the $100 \%$ file sample. Mapping demonstrates that Connecticut is well-represented by the $5 \%$ file in all counties in the state, while New Jersey is generally under-represented by the $5 \% \mathrm{SAF}$. In other states, some counties are over- and others under-represented, and patterns change over time (2001-2003).

Of policy importance, the covariates that produce discrepancies in regression are several that are modifiable by policy: assistance to elderly in purchasing Part B premiums through state buy-in programs; supply of oncologists; rural intensity; and Medicare managed care penetration. Although our findings are not likely to represent all of the United States, they do provide some cause for concern, especially when interpreting results from disparities research or when smallarea analysis of access to facilities are based on $5 \%$ samples.

\section{Acknowledgments}

This work was supported by a National Cancer Institute grant (1Ro1CA126858) and an American Recovery and Reinvestment Act (ARRA) supplement to it. The content is solely the responsibility of the authors and does not necessarily represent the official views of RTI International, the National Cancer Institute, or the National Institutes of Health.

\section{References}

Aranda, J M, Johnson, J W and Conti J B.: Current trends in heart failure readmission rates, Clin. Cardiol. V 32, number 1, (2009): pp 47-52

Congressional Budget Office (CBO), "Geographic Variation in Health Care Spending", February 2008, available online July 14, 2010:

http://www.cbo.gov/ftpdocs/89xx/doc8972/02-15GeogHealth.pdf

Connor, S Elwert, F, Spence, C, and Christakis, N, "Geographic Variation in Hospice Use in the United States in 2002", Journal of Pain and Symptom Management, Vol. 34 No. 3 September 2007: pp 277285 .

Connor SR, Elwert F, Spence C, Christakis NA.," Racial disparity in hospice use in the United States in 2002", Palliat Med. 2008 Apr;22(3):205-13.

Dillavou, E., Muluk, S., Makaroun, M., "A decade of change in abdominal aortic aneurysm repair in the United States: Have we improved outcomes equally between men and women?", Journal of Vascular Surgery, Volume 43, Number 2, February 2006: pp 230-238

Goodman DC, Mick SS, Bott D, Stukel T, Chang C, Marth N, Poage J, Carretta HJ. Primary care service areas: A new tool for the evaluation of primary care services. Health Services Research. 2003;38(1):287310.

Hernandez AF, Whellan DJ, Stroud S, Sun JL, O'Connor CM, Jollis JG., "Outcomes in heart failure patients after major noncardiac surgery", $J$ Am Coll Cardiol. 2004 Oct 6;44(7):1446-53.

Hogan, C, Lunney, J, Gabel, J and Lynn, J, "Medicare Beneficiaries' Costs Of Care In The Last Year Of Life", Health Affairs, v 20, number 4, July/August 2001: pp 188-195

Jaff MR, Cahill KE, Yu AP, Birnbaum HG, Engelhart LM., "Clinical outcomes and medical care costs among 
medicare beneficiaries receiving therapy for peripheral arterial disease", Ann Vasc Surg. 2010 Jul;24(5):57787.

Koroukian SM, Xu F, Dor A, Cooper GS., "Colorectal cancer screening in the elderly population: disparities by dual Medicare-Medicaid enrollment status", Health Serv Res. 2006 Dec;41(6):2136-54.

Koroukian, S. Litaker, D., Dor, A., Cooper, G.. "Use of Preventive Services by Medicare Fee-For-Service Beneficiaries Does Spillover From Managed Care Matter?”, Medical Care, v 43 (5)(2005): pp 445-452.

MedPAC. June 2004. Report to Congress: New approaches in Medicare. Dual eligible beneficiaries: an overview (Chapter 3). Available at: http://www_medpac.gov/documents/Juneo4_Entire_ Report.pdf. Accessed September 18, 2008.

Mobley, L. Root, E, Anselin, L, Lozano-Gracia, N and Koschinsky, J, (2006). "Spatial Analysis of Elderly Access to Primary Care Services." International Journal of Health Geographics, Vol. 5., epub May 15, 2006: http://www.ij-healthgeographics.com/content/ 5/1/19; PMCID: PMC1482683

Mobley, L.R., T. Kuo, and L.S. Andrews. "How Sensitive are Multilevel Regression Findings to Defined Area of Context? A Case Study of Mammography Use in California." Medical Care Research and Review, v 65 (June 1, 2008a), pp 315337; PMID: 18259047

Mobley, L., Kuo, T., Driscoll, D., Clayton, L., and Anselin, L., "Heterogeneity in Mammography Use Across the Nation: Separating Evidence of Disparities from the Disproportionate Effects of Geography", International Journal of Health Geographics. 30 June 2008b, 7:32 available online: http://www.ijhealthgeographics.com/content/7/1/32;

PMID: 18590540

Mobley, L., Subramanian, S., Koschinsky, J., Frech, H.E., Clayton, L., and Anselin, L. "Medicare managed care penetration and diffusion of colonoscopy among FFS Medicare", Health Services Research, Vol 46, Issue 6 (2011), pp 1905-1927.

Mobley, L., Kuo, M., Urato, M., Boos, J., Lozano-
Gracia, N., and Anselin, L., "Predictors of Endoscopic Colorectal Cancer Screening over Time in 11 States", Cancer Causes and Control, Volume 21, Issue 3 (2010), Page 445-461.

Niefeld, M, Braunstein, J, Wu, A, Saudek, C, Weller, W, and Anderson, G, "Preventable Hospitalization Among Elderly Medicare Beneficiaries With Type 2 Diabetes", Diabetes Care, v 26, number 5, May 2003: pp 1344-1349.

Pearlman AS, Ryan T, Picard MH, Douglas PS.," Evolving trends in the use of echocardiography: a study of Medicare beneficiaries", J Am Coll Cardiol. 2007 Jun 12;49(23):2283-91.

Pyenson, B, Connor, S, Fitch, K and Kinzbrunner, B, "Medicare Cost in Matched Hospice and Non-Hospice Cohorts", Journal of Pain and Symptom Management, Vol. 28 No. 3 September 2004: pp 200210.

Rao, V. M., Parker, L., Levin, D. C., Sunshine, J., Bushee, G. (2001). Use Trends and Geographic Variation in Neuroimaging: Nationwide Medicare Data for 1993 and 1998. Am. J. Neuroradiol. 22: 16431649

Ryan J, Linde-Zwirble W, Engelhart L, Cooper L, Cohen DJ., "Temporal changes in coronary revascularization procedures, outcomes, and costs in the bare-metal stent and drug-eluting stent eras: results from the US Medicare program", Circulation. 2009 Feb 24;119(7):952-61. Epub 2009 Feb 9.

SAS (2010) Base SAS® 9.2 Procedures Guide: Statistical Procedures, Third Edition, Available online July 2010: http://support.sas.com/documentation/ cdl/en/procstat/63104/HTML/default/viewer.htm\# documentation/cdl/en/procstat/63104/HTML/defau lt/procstat freq a0000000658.htm

Strutton, D., and Walt, J., "Trends in Glaucoma Surgery before and after the Introduction of New Topical Glaucoma Pharmacotherapies", Journal of Glaucoma, Volume 13, Number 3, June 2004: pp 221226

Wennberg JE., "Understanding geographic variations in health care delivery", $N$ Engl J Med. 1999 Jan $7 ; 340(1): 52-3$ 